\title{
Astroparticle physics with ARGO-YBJ
}

\author{
Paolo Camarri*i \\ Università degli Studi di Roma "Tor Vergata" and INFN Roma Tor Vergata \\ E-mail: paolo.camarri@roma2.infn.it
}

The ARGO-YBJ experiment was installed in the Tibet region of China, 4300 meters above sea level. It run continuously from November 2007 until February 2013, with the goal of observing astronomical $\gamma$-ray sources in the energy range between a few hundred $\mathrm{GeV}$ and about $100 \mathrm{TeV}$, and primary cosmic rays in the energy range between about $1 \mathrm{TeV}$ and a few PeV. The unique feature of the ARGO-YBJ detector was its full-coverage layout of Resistive Plate Chambers on an area of $(78 \times 74) \mathrm{m}^{2}$, with a guard ring around and a full area of $11000 \mathrm{~m}^{2}$. The most important results obtained by ARGO-YBJ will be presented, with specific focus on the observation and monitoring of galactic and extragalactic $\gamma$-ray sources and primary light-nuclei spectrum and knee.

The European Physical Society Conference on High Energy Physics

5-12 July, 2017

Venice

* Speaker.

${ }^{\dagger}$ on behalf of the ARGO-YBJ Collaboration 


\section{Introduction}

The ARGO-YBJ experiment [1] was a collaboration between Chinese and Italian research groups. I was installed in Tibet, China, at an altitude of $4300 \mathrm{~m}$ a.s.l. in order to reconstruct air showers generated by cosmic rays with energy down to a few hundred GeV. ARGO-YBJ had been designed to investigate a large number of topics in astrophysics and cosmic-ray physics: $\gamma$-ray astronomy (search for point-like sources above few hundreds of $\mathrm{GeV}$ ), search for VHE tails of $\gamma$-ray bursts above $\sim 1 \mathrm{GeV}$, cosmic-ray physics, Sun and heliosphere physics. The ARGO-YBJ detector was based on a full-coverage single layer of Resistive Plate Chambers (RPCs) covering a surface of $74 \times 78 \mathrm{~m}^{2}$. A group of 12 neighboring RPCs was called a "cluster", and the fullcoverage part of the detector included 130 clusters. Around this, 23 additional clusters were placed in order to obtain a better reconstruction of the shower front, which extended the experiment area to $110 \times 100 \mathrm{~m}^{2}$. The space-time unit of the ARGO-YBJ detector was a "pad", namely a group of 8 neighboring RPC read-out strips. The single-hit time resolution was about 1.8 ns [2]. Air showers were triggered by requiring a number of hits greater than 20 within a $150 \mathrm{~ns}$ time window, giving an average trigger rate of about $3.5 \mathrm{kHz}$ with a dead time of $4 \%$ and an average duty cycle greater than $86 \%$. ARGO-YBJ had been running almost uninterruptedly with its complete layout since October 2007 with a duty cycle of $90 \%$ and an average trigger rate of $3.6 \mathrm{kHz}$. A selection of its main physics results is presented here, focusing on the monitoring of $\gamma$ rays from astromomical sources and the "light" cosmic-ray spectrum up to about $1 \mathrm{PeV}$.

\section{Monitoring of galactic and extra-galactic $\gamma$-ray sources with ARGO-YBJ}

In ARGO-YBJ, the $\gamma$-ray flux from point-like or extended sources could only be measured by subtraction from the cosmic-ray background, by using the well-established "time-swapping" and "equi-zenith" methods. The results were carefully checked by monitoring a well known galactic $\gamma$-ray source, the Crab nebula, in the energy region above a few hundred $\mathrm{GeV}$, as shown in figure 1 [2].

The analysis of the whole ARGO-YBJ data set gave a statistical significance above 5 standard deviations for $6 \gamma$-ray sources in the above-mentioned energy region. Extremely noticeable results were obtained for the $\gamma$-ray emission of two AGN: Markarian 421 (MRK421) and Markarian 501 (MRK501).

Figure 2 shows the integral $\gamma$-ray flux from MRK421 over a time span of more than 3 years as measured by ARGO-YBJ and by two other satellite experiments in different energy regions, with the vertical axes suitably rescaled. A few remarkable flares from the AGN MRK421 were observed in the X-ray range by the ASM X-ray telescope in 2006, 2008 and 2010, and ARGO could observe $\mathrm{TeV} \gamma$ emission at the same time for such flares, as shown in figure 2 for the two most recent ones. Concerning the two flares observed on June 4-6 and June 11-13, 2008 [3], ARGO-YBJ investigated the $\gamma$ emission from the same source on those days [4]. The peak significance for the flare of June 11-13, 2008 was 4.2 standard deviations. The ARGO-YBJ data fully satisfy the relation between the spectral index and the flux resulting from the Whipple measurements of a similar flare of MRK421 [5], suggesting that this relation is an intrinsic property of the source. One more remarkable flare from MRK421 occurred on February 2010. ARGO-YBJ observed 


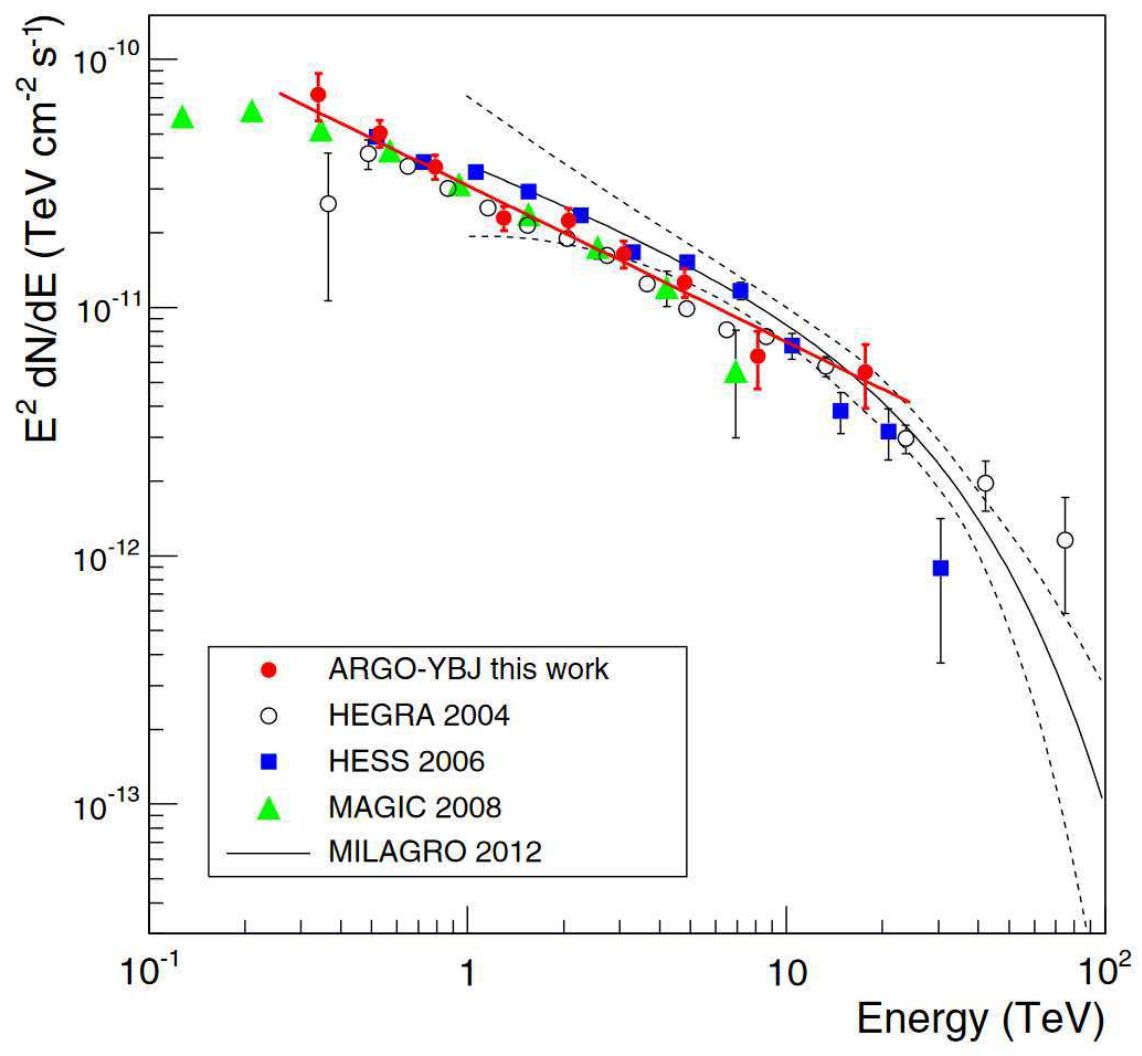

Figure 1: Crab Nebula differential energy spectrum multiplied by $E^{2}$, measured by ARGO-YBJ and other experiments. The thick red solid line represents the best fit of the ARGO-YBJ data. The dotted lines delimit the 1 sigma error band of the Milagro spectrum.

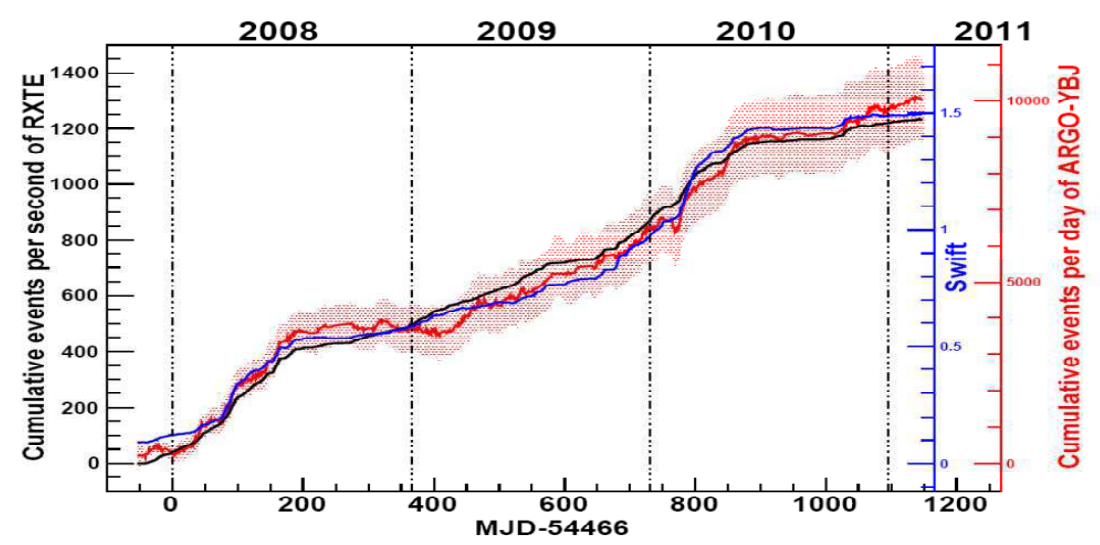

Figure 2: Comparison of the cumulative counting rates for the $\gamma$-ray emission from MRK421 as measured by ARGO-YBJ (red), RXTE/AMS (black) and SWIFT (blue). The vertical coordinates have been rescaled suitably in order to obtain a direct visual comparison of the three experimental results. 
it between February 16th and February 18th at a 6 s.d. significance level. The measured flux exceeded $3 \mathrm{Crab}$ units for the duration of the observation. The importance of this measurement by ARGO-YBJ lies in the fact that for the first time a ground-based experiment could detect a flare with a 5 standard-deviation significance level on a daily basis.

An X-ray flare from the AGN MRK501 was detected by the RXTE/ASM telescope in October 2011 after a long "quiet" period (the previous intense X-ray flare dated back to 1997). This flare was associated to a strong emission in the TeV energy range detected by ARGO-YBJ [6]. During the flaring period, the $\mathrm{TeV}$ emission was observed in 36 days with a significance greater than 6 standard deviations.

\section{Cosmic-ray physics and light-component knee with ARGO-YBJ}

Several results in cosmic-ray physics with ARGO-YBJ were already reported in the past, with particular emphasis on the cosmic-ray flux anisotropy at $\mathrm{TeV}$ energies [7], the study of the moon shadow [8] and the corresponding limit on the $\bar{p}-p$ flux ratio [9], the proton-air interaction cross section [10] and the light-component spectrum up to $200 \mathrm{TeV}$ [11].

Subsequently, a joint analysis of the data collected by ARGO-YBJ and the WFCT-02 Cherenkov telescope placed close to the ARGO-YBJ experimental hall was performed. The data collected by the two detectors from December 2010 and February 2012 were considered, requiring a coincidence within an $8-\mu$ s time window using the GPS event time stamps of the two detectors. The event-selection requirements for ARGO-YBJ were the following: well reconstructed shower-core position, shower direction within $6^{\circ}$ of the telescope axis, more than 6 fired pixels in the telescope PMT matrix, more than 1000 fired pads in the ARGO-YBJ central carpet; the coincident events for the light-component selection were chosen according to the number of charged particles in the shower core and the shape of the Cherenkov-light image.

Figure 3 shows the ARGO-YBJ light-component spectrum (both at lower energy [13] and higher energy [14]) in comparison with the results of other experiments. A steepening of the lightcomponent energy spectrum is observed, starting at about $700 \mathrm{TeV}$. This is compatible with the results previously obtained by Tibet AS- $\gamma$, CASA-MIA and MACRO. This result strongly encourages further investigation in future experiments (e.g. LHAASO), in order to improve our understanding of proton acceleration by SNRs up to energies approaching $1 \mathrm{PeV}$.

\section{Conclusions}

The ARGO-YBJ experiment ran data almost uninterruptedly with its complete layout for more than 5 years, from November, 2007 till February, 2013. Its results in galactic and extragalactic $\gamma$ ray astronomy, and in cosmic-ray physics as well at the energy scale from a few hundred $\mathrm{GeV}$ up to a few PeV, strengthened our knowledge about the $\gamma$-ray emission of AGN and prepared the path for future, deeper investigations of cosmic-ray physics around the knee.

\section{References}

[1] G. Aielli et al. (the ARGO-YBJ collaboration); NIM A 562 (2006) 92-96. 


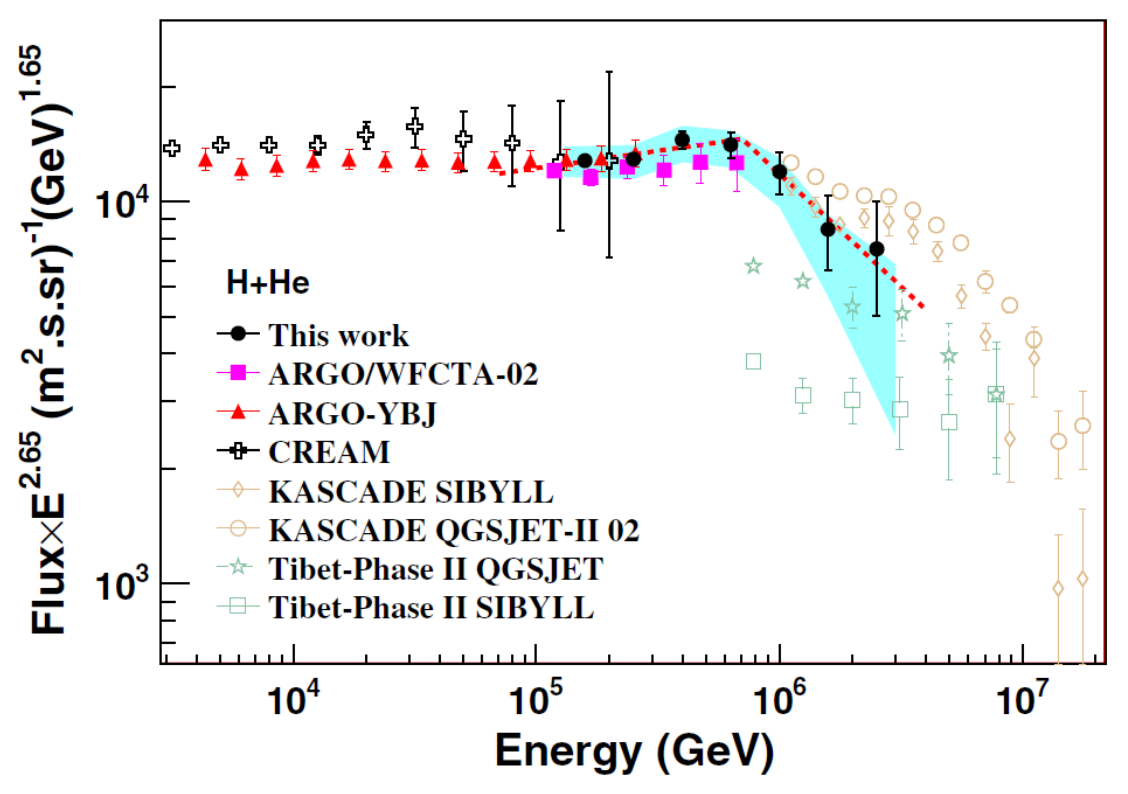

Figure 3: Light-component cosmic-ray spectrum obtained by the hybrid experiment with ARGO-YBJ and the imaging Cherenkov telescope [12]. A clear knee structure is observed around $700 \mathrm{TeV}$. The lightcomponent spectra by CREAM, ARGO-YBJ and the hybrid experiment below the knee, the spectra by Tibet AS- $\gamma$ and KASCADE above the knee are shown for comparison. In our result, the error bar is the statistical error, and the shaded area represents the systematic uncertainty.

[2] B. Bartoli et al. (the ARGO-YBJ collaboration); ApJ 798 (2015) 119.

[3] I. Donnarumma et al.; ApJ 691 (2009) L13-L19.

[4] G. Aielli et al. (the ARGO-YBJ collaboration); ApJ Letters 714 (2010) L208-L212.

[5] F. Krennrich et al. (the WHIPPLE collaboration); ApJ 575 (2002) L9-L13.

[6] B. Bartoli et al. (the ARGO-YBJ collaboration); ApJ 758 (2012) 2.

[7] B. Bartoli et al. (the ARGO-YBJ collaboration); Phys. Rev. D 88 (2013) 082001.

[8] B. Bartoli et al. (the ARGO-YBJ collaboration); Phys. Rev. D 84 (2011) 022003.

[9] B. Bartoli et al. (the ARGO-YBJ collaboration); Phys. Rev. D 85 (2012) 022002.

[10] G. Aielli et al. (the ARGO-YBJ collaboration); Phys. Rev. D 80 (2009) 092004.

[11] B. Bartoli et al. (the ARGO-YBJ collaboration); Phys. Rev. D 85 (2012) 092005.

[12] B. Bartoli et al. (the ARGO-YBJ collaboration); Phys. Rev. D 92 (2015) 092005.

[13] B. Bartoli et al. (the ARGO-YBJ collaboration); Phys. Rev. D 91 (2015) 112017.

[14] B. Bartoli et al. (the ARGO-YBJ collaboration); Chin. Phys. C 38 (2014) 045001. 